\title{
Reliability oriented optimum design of the Large Hadron Collider magnet-to-magnet interconnections
}

\author{
Cédric Garion $^{1, a}$, Didier Marquis ${ }^{2}$ and Blazej Skoczen ${ }^{1}$ \\ 1 CERN, LHC Division, 1211 Geneva 23, Switzerland \\ 2 IFMA, Campus des Cézeaux, BP 265, 63175 Aubières Cedex, France
}

Received 3 September 2002, accepted le 23 October 2003

\begin{abstract}
The quest for high particle energies of modern super conducting lepton and hadron storage rings assumes a strong minimisation of the non-magnetic interconnection zones when compared to the total magnetic length of the main quadrupoles and the dipoles. The ratio of non-magnetic to magnetic zones in the LHC is close to $3 \%$. Such a low percentage leads to a very compact design of systems and sub-systems situated in the interconnections, like the mechanical compensation system, composed of the bellows expansion joints, that have to compensate for the thermal contraction of the magnets. Given the complexity of the LHC interconnections, the requirements concerning their reliability were considerably increased since the availability of the collider for physics cannot be compromised. The failure modes taken into account in the present analysis are grouped into 3 categories: material failures, structural stability failures and fatigue failures. Optimisation of stainless steel for cryogenic applications has been carried out. Also, a concept of reliability oriented parametric optimisation of the LHC interconnections is presented.
\end{abstract}

Key words: Bellows expansion joints / cryogenic temperatures / reliability / damage / martensitic transformation / fatigue / stability / Weibull

Résumé - Conception optimale, orientée vers la fiabilité des interconnexions des aimants du Large Hadron Collider. La recherche sur les anneaux supraconducteurs de stockage de leptons et hadrons, pour de hautes énergies de particules, suppose une forte minimisation de la longueur des zones non magnétiques des interconnexions, comparée à la longueur magnétique totale des dipôles et quadripôles principaux. Le rapport des zones non-magnétiques et magnétiques est proche de $3 \%$. Un pourcentage si faible conduit à une conception très compacte des systèmes et sous-systèmes situés dans les interconnexions, tels que le système de compensation mécanique, composé de soufflets d'expansion, qui doivent compenser les contractions thermiques des aimants. Étant donnée la complexité des interconnexions du LHC, les contraintes concernant leur fiabilité ont été considérablement augmentées puisque la disponibilité du collisionneur pour les expériences ne peut être compromise. Les modes de rupture pris en compte dans cette analyse sont groupés en 3 catégories : rupture du matériau, instabilité structurale et rupture par fatigue. L'optimisation d'un acier inoxydable pour des applications cryogéniques a été réalisée. Un concept de fiabilité orientée vers l'optimisation paramétrique des interconnexions du LHC est également présenté.

Mots clés : Soufflets de compensation / températures cryogéniques / fiabilité / endommagement / transformation martensitique / fatigue / stabilité / Weibull

\section{Introduction}

The modern high-energy physics needs very sophisticated and complex tools in order to explore the world of elementary particles constituting the matter. One of the most important aims over the past 30 years was confirmation of the so-called Standard Model which assumes that

\footnotetext{
${ }^{a}$ Corresponding author: cedric.garion@cern.ch
}

the fundamental constituents of matter form three families of quarks and leptons. The relevant scientific tools are called accelerators, storage rings and colliders and their main function is to produce, accelerate, store and collide the beams of particles in order to search for the new elementary events, announcing the potential discoveries, and to provide more statistics for the already known reactions.

One of the fundamental principles of the superconducting accelerators is the principle of maximum of 


\section{Nomenclature}

\begin{tabular}{|lll|}
\hline$\nu$ & Poisson' coefficient & \\
$\mu$ & Magnetic permeability & \\
$\xi$ & Volume fraction of martensite & \\
$\mu_{f}$ & Correction factor & \\
$\Delta \varepsilon_{p}$ & Plastic strain range & \\
$\varepsilon_{f 0}$ & Tensile ductility & \\
$\xi_{L}$ & Saturation level of martensitic transformation \\
$\varepsilon_{p m}$ & Mean plastic strain & \\
$A$ & Phase transformation intensity parameter & \\
$C_{f}$ & Correction factor & \\
$D$ & Damage parameter & $\mathrm{m}$ \\
$D_{m}$ & Bellows mean diameter & $\mathrm{m}$ \\
$D_{i n}$ & Inner diameter & $\mathrm{m}$ \\
$D_{o u t}$ & Outer diameter & $\mathrm{N} \cdot \mathrm{m}^{-2}$ \\
$E_{T}$ & Young' modulus at the temperature $T$ & $\mathrm{~N} \cdot \mathrm{m}^{-1}$ \\
$F_{a x}$ & Axial tangent stiffness & $\mathrm{m}$ \\
$h$ & Wall thickness & $\mathrm{m}$ \\
$L_{b}$ & Length of bellows (convolutions) & $\mathrm{N} \cdot \mathrm{m}^{-1}$ \\
$N_{\theta}$ & Normal circumferential stress & $\mathrm{N} \cdot \mathrm{m}^{-1}$ \\
$N_{\Phi}$ & Normal meridional stress & \\
$n_{c}$ & Number of convolutions \\
$N_{f}$ & Number of cycles to failure & \\
$n_{p}$ & Number of plies & $\mathrm{N}$ \\
$P$ & Axial force & $\mathrm{N}$ \\
$p$ & Accumulated plastic strain & $\mathrm{m}$ \\
$p_{\xi}$ & Phase transformation threshold & $\mathrm{m}$ \\
$p_{D}$ & Damage threshold & $\mathrm{N} \cdot \mathrm{m}^{-2}$ \\
$R$ & Reliability & \\
$r$ & Curvature radius & \\
$S$ & Strength energy of damage \\
$T$ & Temperature & \\
$t_{p}$ & Thickness of ply \\
$w$ & Convolution depth & \\
$x$ & Axial displacement & \\
$Y$ & Strength energy density release rate & \\
& & \\
\hline
\end{tabular}

magnetic length. The ratio between the length of arc with the presence of magnetic field and the accumulated length of magnet-to-magnet interconnections has to be maximised. To achieve the maximum beam energy in the LHC (Large Hadron Collider being at present constructed at CERN) the accumulated length of spatial zones, dedicated to the interconnections between the main cryomagnets, has been limited to $3 \%$ of the total magnetic length in the Arcs and Dispersion Suppressors. This implies an extremely compact design of systems and subsystems located in the interconnections [1] (Fig. 1) like: bellows expansion joints, splices of the superconducting bus-bars, RF contacts (Radio-Frequency gold coated copper fingers) etc.

The compactness has been enhanced by the two-inone specific magnet configuration in the LHC. In order to cope with the space limitations a deliberate choice, supported by a mathematical optimisation, was made and the LHC bellows expansion joints work in highly inelastic regime called low-cycle fatigue. All deformation developed during cool-down and warm-up of the collider is localised in the interconnections (the accumulated value

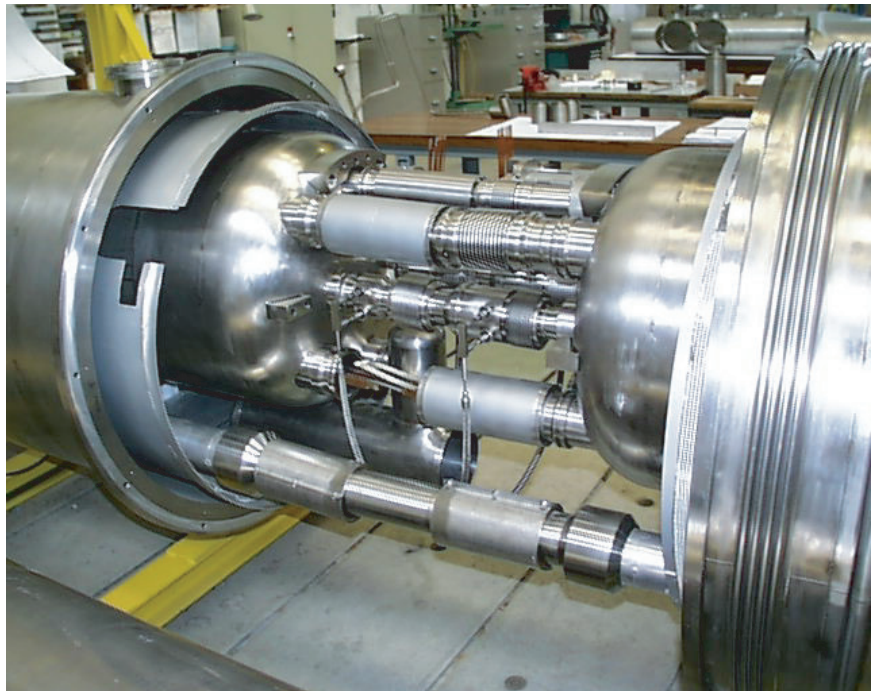

Fig. 1. LHC interconnections.

of thermal contraction approaches 70 meters for the LHC Arcs and DS zones). In the past the requirements imposed on the compensation elements were less stringent. The accelerators and their interconnections operated at ambient temperature (for example Large Electron-Positron at CERN). Therefore, a standard compensation between the beam line portions was sufficient. The LHC requires much more sophisticated compensation systems that fulfil all the functions both at room and at sub-zero temperatures $(1.9 \mathrm{~K})$. Thus, the requirements concerning the reliability of the LHC interconnections were strongly increased.

In the present paper a concept of reliability oriented parametric optimisation of the LHC compensation system (bellows expansion joints) is presented. A global availability of the LHC interconnections is defined at the level of $99.5 \%$. It is based on the assumption of maximum one short intervention (10.5 days) over 10 years of LHC operation (200 days a year) due to a failure in one of the interconnection systems.

\section{Mechanical compensation systems of the superconducting accelerators}

The zones of interconnections in a modern superconducting accelerator of particles (Fig. 1) are the zones where the deformation of the structure due to thermomechanical cycles is localised. Indeed, the contraction of magnets when cooling down and their expansion when warming up are entirely compensated by the expansion joints. The highly optimised expansion joints dissipate a considerable amount of plastic power over a single thermo-mechanical cycle. Therefore, their fatigue life stays usually between 500 and 5000 cycles to failure (low cycle fatigue).

The compensation systems used for low temperature interconnections are based on expansion joints of the same type as those applied at room and at high temperatures 


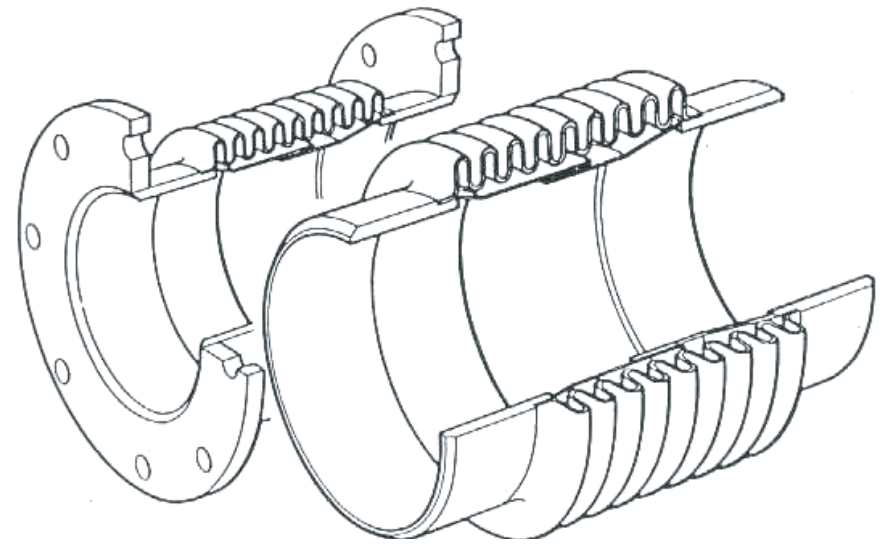

(a)

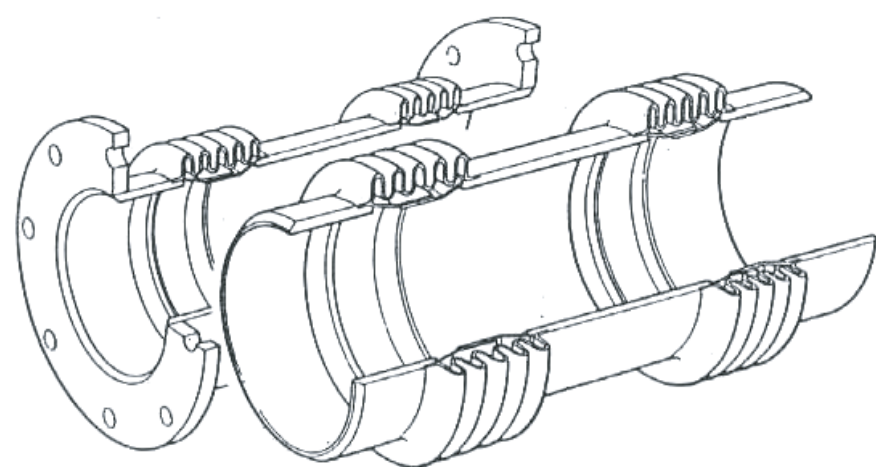

(b)

Fig. 2. (a) U-type single bellows expansion joints with internal protection. (b) U-type universal joints (double bellows) with internal protection.

(see Figs. 2a and 2b). The expansion joints work often in extremely severe service conditions comprising temperature variations between ambient and operational conditions $(1.9 \mathrm{~K})$, high internal pressure (up to 20 bars), large cyclic axial offset (of the order of the bellows length), different types of misalignment offsets $(4-5 \%$ of the bellows length) as well as irradiation (1 kGy/year). They are supposed to compensate for mismatch of dimensional tolerances and they should provide leak-tight transitions between the adjacent components (magnets, pressure or vacuum vessels). The minimum fatigue life specified in the optimisation process is 500 cycles (low-cycle fatigue) and the target value for the analysis of reliability is equal to 50 cycles.

Typical expansion joints applied at cryogenic temperatures are:

- single U-type, multi-ply bellows and universal expansion joints (cryogenic transfer lines, accelerator interconnections);

- Special very compact thin-walled expansion joints (nested bellows, edge welded bellows for vacuum applications).

All of them are made of materials easy to form plastically and showing high level ductility under cryogenic conditions (mainly stainless steels). Usually, they can accumulate a large plastic deformation (due to low-cycle fatigue and ratchetting) before the process of macro-crack propagation starts.

\subsection{Standard expansion joints for interconnections between helium enclosures}

Active part of the standard expansion joint is a corrugated axisymmetric membrane having usually a U-type profile of convolutions. These thin-walled shells are manufactured out of seamless or longitudinally welded cylinders, often heat treated in order to make the process of mechanical forming sufficiently easy. The expansion joints designed for high internal or external pressure applications are usually manufactured as multiply shells. Such a structure is characterised by a low axial stiffness and, at the same time, by a high pressure capacity of the bellows. One of the main problems when forming multiply bellows is the interlayer friction and possible damage of the surfaces staying in contact. The single bellows (Fig. 2a) are used as compensation units in the interconnections between the magnets where alignment movements are small. The transverse offset of these units is rather limited. On the other hand, the universal joints (Fig. 2b) are applied in the peripheral lines, where considerable transverse movements are required. Universal joints ( 2 single bellows linked by an intermediate tube), offering less stiffness with respect to lateral movements, are simultaneously less stable under inner pressure and require special methods to predict the buckling behaviour.

One of the most important parameters contributing to stability of interconnections is the axial stiffness of expansion joints. The axial stiffness is defined either as the elastic tangent stiffness measured when unloading the shell:

$$
F_{a x}^{t}=\frac{\mathrm{d} P}{\mathrm{~d} x}
$$

or as the elastic-plastic secant stiffness:

$$
F_{a x}^{s}=\frac{\Delta P}{\Delta x}
$$

both are based on the hysteresis curves for the locally plastified convolutions. Here $P, x$ denote the axial force and the corresponding displacement, respectively. Typical hysteresis loops computed for the same expansion joint with U-type convolutions, corresponding to three temperature levels: $293 \mathrm{~K}$ (room temperature), $77 \mathrm{~K}$ (liquid nitrogen) and $4 \mathrm{~K}$ (liquid helium), are shown in Figure 3 (cf. [2]). Due to evolution of the material properties, the hysteresis loops at low temperatures show much smaller amount of plastic deformation than at room temperature. Thus, the total amount of plastic power dissipated in the convolutions when closing a complete loop turns out to be smaller. This leads to increase of fatigue life at cryogenic temperatures, which is a direct consequence of decrease of the energy dissipated over a stabilised cycle. 


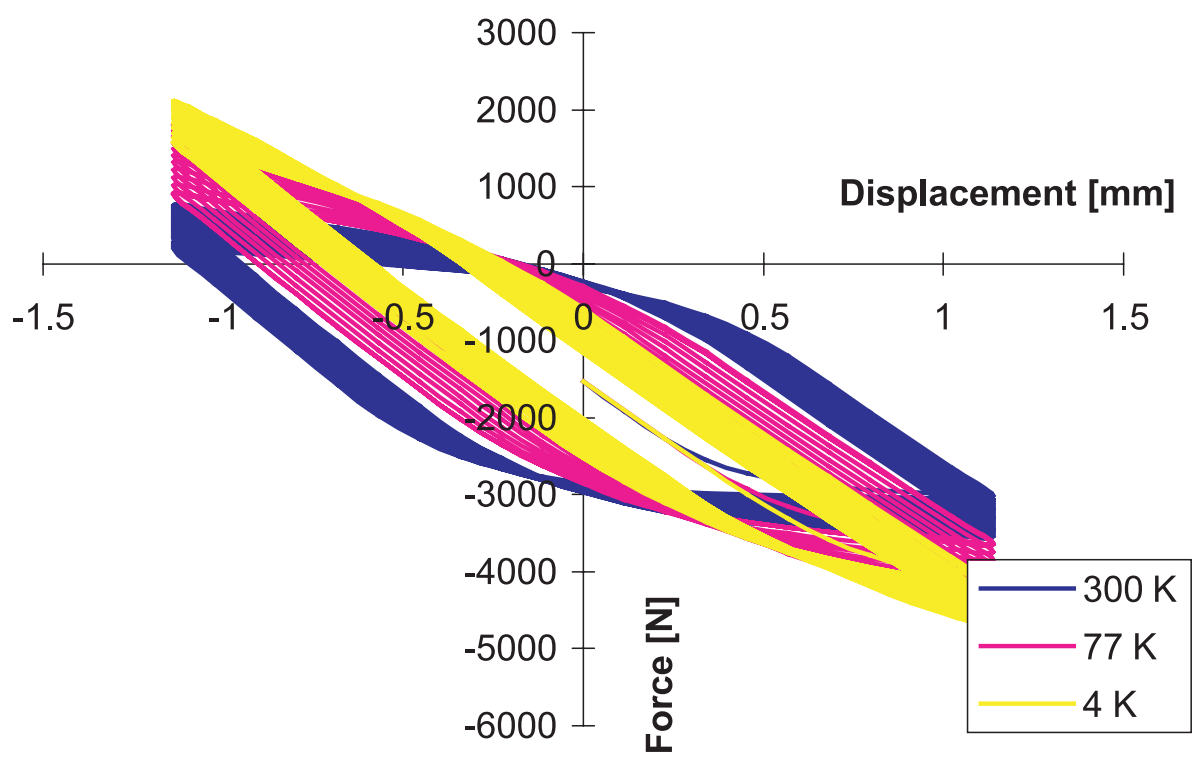

Fig. 3. Hysteresis loops of expansion joint computed for three temperature levels (room and cryogenic temperatures).

Table 1. Evolution of the elastic axial stiffness with temperature.

\begin{tabular}{ccc}
\hline Temperature & $\begin{array}{c}\text { Modulus of } \\
\text { Elasticity }\end{array}$ & $\begin{array}{c}\text { Bellows elastic axial } \\
\text { stiffness }\end{array}$ \\
{$[\mathrm{K}]$} & $E_{T}$ & $F_{a x / T}^{t}$ \\
\hline 293 & $E_{293}$ & $F_{a x / 293}^{t}$ \\
77 & $E_{77} \cong 1.08 E_{293}$ & $F_{a x / 77}^{t}=1.08 F_{a x / 293}^{t}$ \\
4 & $E_{4} \cong 1.08 E_{293}$ & $F_{a x / 4}^{t}=1.08 F_{a x / 293}^{t}$ \\
\hline
\end{tabular}

The bellows axial stiffness, as determined by the Expansion Joint Manufacturers Association (EJMA, 1993; 1995), can be expressed by the following formula:

$$
F_{a x}=\frac{\mu_{f}}{C_{f}}\left(E_{T} D_{m}\left(\frac{t_{p}}{w}\right)^{3}\left(\frac{n_{p}}{n_{c}}\right)\right)
$$

where $E_{T}$ denotes the modulus of elasticity at the temperature $T, D_{m}$ stands for the bellows mean diameter, $t_{p} / w$ is the thickness of ply to convolution depth ratio, $n_{p} / n_{c}$ is the number of plies to number of convolutions ratio and $\mu_{f}, C_{f}$ are the correction factors. Thus, the elastic axial stiffness is directly proportional to the modulus of elasticity at a given temperature. It is worth pointing out that the axial stiffness is proportional to the third power of the ply thickness and inversely proportional to the third power of the convolution depth. Both parameters are fundamental for the optimisation process (reduction of the axial stiffness). Evolution of the axial stiffness as a function of temperature for expansion joints made of 316L stainless steel is shown in Table 1.

Both at the temperature of liquid helium $(4 \mathrm{~K})$ and at the temperature of liquid nitrogen $(77 \mathrm{~K})$ the elastic axial stiffness is approximately $8 \%$ higher than at room temperature. Hence, the expansion joint turns out to be stiffer. The elastic-plastic axial stiffness is a function of the total axial offset imposed on the expansion joint. Generally, larger offset leads to higher amount of plastic strains in the bellows convolutions and, consequently, to smaller secant stiffness. This parameter can be measured between the points of return on the stabilised hysteresis loops for a given temperature. In the equation for the bellows axial stiffness (Eq. (3)) the correction factor $\mu_{f}$ can be expressed as:

$$
\mu_{f}=\mu_{f}\left(\Delta \varepsilon^{p}, T\right),
$$

since it is a function of the plastic strain range $\Delta \varepsilon^{p}$ on a stabilised cycle and the temperature $T$.

\subsection{Non-standard expansion joints for beam vacuum interconnections}

The particle beams circulate in the channels connected by means of very thin, corrugated shells (typical thickness of $0.15 \mathrm{~mm}$ ) which separate ultra-high beam vacuum from the insulation vacuum. The thickness to depth of convolution $\left(\frac{h}{w}\right)$ and the thickness to pitch $\left(\frac{h}{q}\right)$ ratios are 0.0184, 0.0291, respectively. In addition, thickness to mean diameter ratio $\left(\frac{h}{D_{m}}\right)$ is equal to $1.66 \times 10^{-3}$. Under normal operating conditions there is no differential pressure between the inside and the outside of the beam lines. Therefore, the design of the relevant compensation units can be particularly "light". Exceptionally, in case the beam or the insulation vacuum is broken, the bellows can be subjected to inner or outer atmospheric pressure. Both situations are taken into account as the load cases. Since the beam vacuum interconnections stay in permanent thermal contact with the helium enclosure (magnets) their operational temperature is usually equal to the temperature of the magnets. Thus, all the problems associated with operation of thin shells at sub-zero temperatures remain valid. 

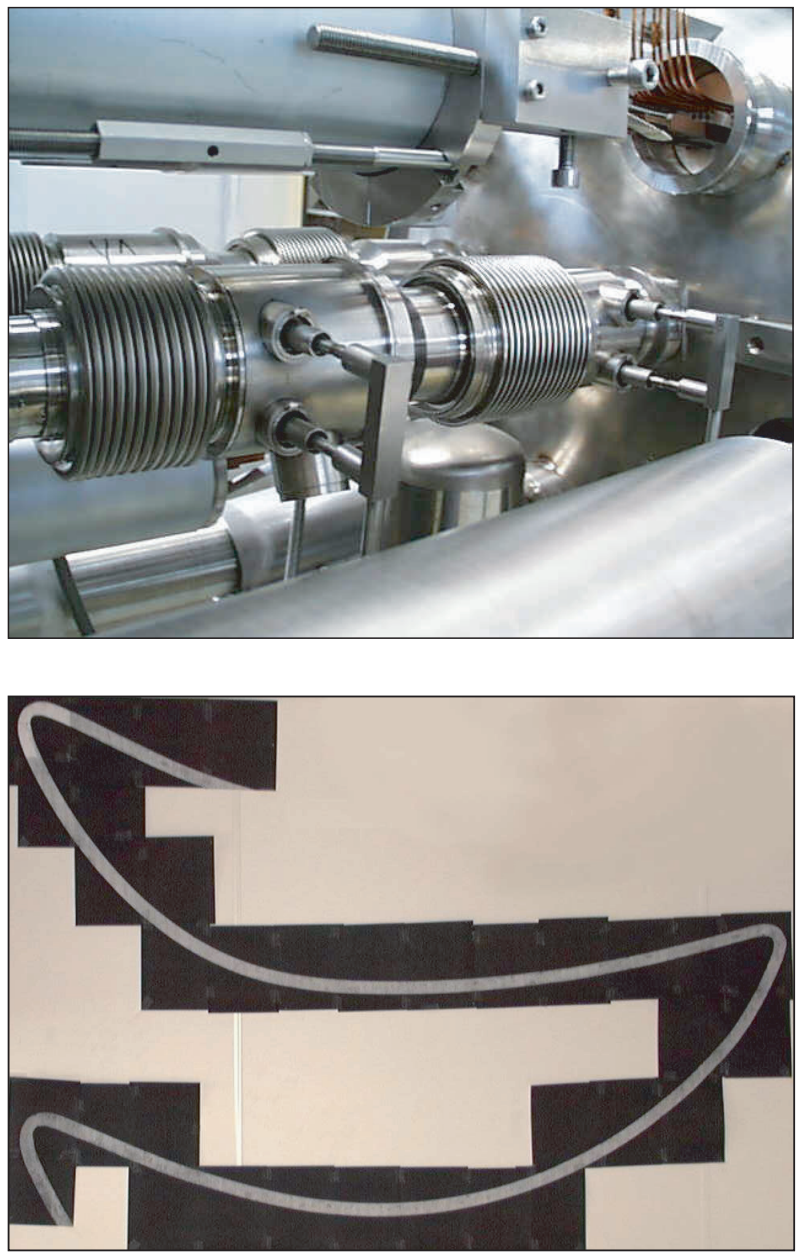

Fig. 4. (a) Beam vacuum interconnect - compact nested bellows on the left (CERN-LHC). (b) Convolution profile of the nested bellows.

The requirement of limited axial space available for installation of interconnections leads to very compact design of the expansion joints. A modern alternative for the beam vacuum interconnections constitute the nested bellows, single ply thin-walled corrugated shells, having a specific "nested" profile of convolutions (Figs. 4 and 4b).

This very compact design leads to an exceptional ratio 1:1 between the compensation capacity and the length of bellows. On the other hand, the fatigue life of these compact units is usually quite low since the bellows operate in the domain of low cycle fatigue. Also, the nested bellows are very sensitive with respect to imperfections (geometrical, material) both from the point of view of fatigue and stability (buckling). Since the compensation units remain in the direct proximity of the particle beams it is important to reduce their magnetic permeability as much as possible in order to avoid creation of periodic beam perturbation fields. Typical initial value of magnetic permeability is 1.005 . However, the magnetic permeability can drastically increase as a result of the plastic strain induced martensitic transformation at cryogenic temperatures. Therefore, it is crucial to choose for the beam
Table 2. Reliability categories.

\begin{tabular}{|c|c|c|c|c|}
\hline \multirow{6}{*}{ 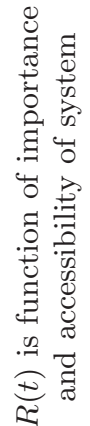 } & Category & $R(t)[\%]$ & Category & $R(t)[\%]$ \\
\hline & 0 & 99.9999 & 5 & 99.999998 \\
\hline & 1 & 99.99995 & 6 & 99.999999 \\
\hline & 2 & 99.99998 & 7 & 99.9999995 \\
\hline & 3 & 99.99999 & 8 & 99.9999998 \\
\hline & 4 & 99.999995 & 9 & 99.9999999 \\
\hline
\end{tabular}

vacuum interconnections the materials showing an exceptional stability at sub-zero temperatures. This particular problem is explained in the next chapter in detail since increase in magnetic permeability of the beam vacuum bellows beyond a predefined value constitutes also a failure mode. Such a failure, specific to the material applied for bellows convolutions, can be classified as material failure (contrary to the structural failure modes like fatigue failure or bellows instability).

\section{Apportionment of the reliability levels to the LHC interconnections}

Since in the LHC interconnections there are 3 main systems that might fail: mechanical compensation system (bellows expansion joints), electrical connections (superconducting bus-bars) and beam image current continuity system (fixed and sliding RF contacts) it is assumed that the expected availability is apportioned to each system on an equal basis. Thus, the expected availability amounts to $99.8 \%$ for either of them. Given the number of interconnections in the LHC Arc and DS zones (1618) the apportioned availability per one interconnect is fixed to $99.9999 \%$. The apportionment of the reliability levels to subsystems and components in each LHC interconnect corresponds to the reliability categories presented in Table 2 .

As an example, allocation of these categories to the standard LHC dipole/dipole interconnect is shown in Table 3. Lower categories (0-4) are reserved for the whole interconnect and the sub-systems whereas the higher categories (5-9) are reserved for the components. For instance, a typical reliability level allocated to the RF-contact module is equal to $99.999999 \%$ (category 6 ).

The redistribution of reliability levels among the components of the mechanical compensation system is non-uniform and follows the above described reliability categories. To this end each LHC interconnection was subdivided into a number of zones, as shown in Figure 5.

The zones reflect the natural arrangement of systems in the interconnections:

- Zone 1 - beam vacuum interconnects (V1, V2);

- Zone 2 - main cryogenic connections (M1, M2, M3); 


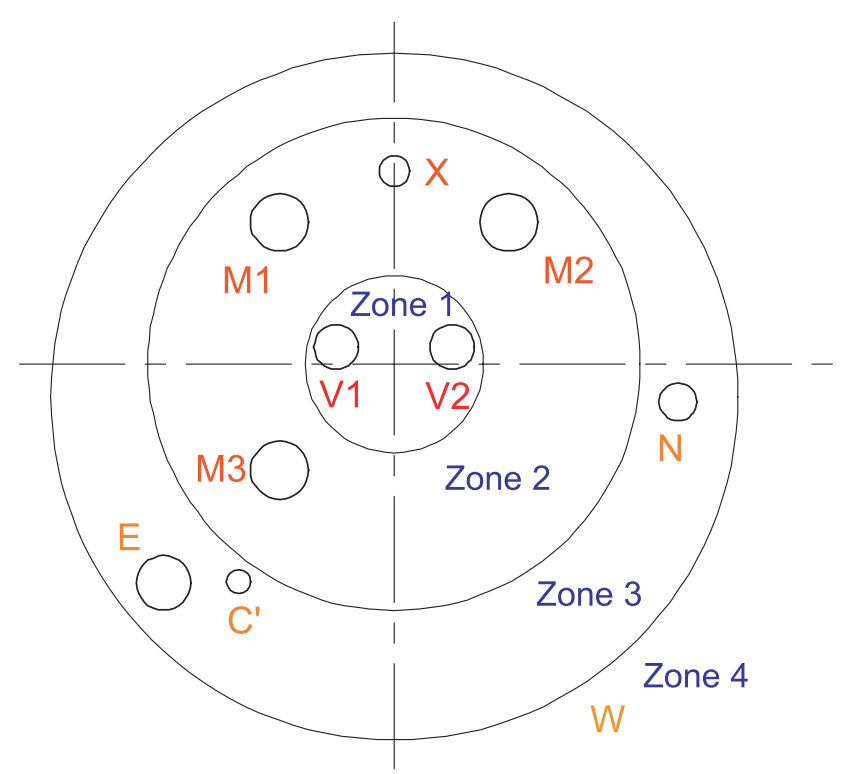

Fig. 5. Reliability zones in the LHC interconnections.

Table 3. Standard LHC magnet-to-magnet interconnect.

\begin{tabular}{llll}
\hline $\begin{array}{l}\text { Sub-system: } \\
\text { interconnection line }\end{array}$ & $\begin{array}{l}\text { Nr of } \\
\text { lines }\end{array}$ & $\begin{array}{l}\text { Reliability } \\
\text { per line [\%] }\end{array}$ & $\begin{array}{l}\text { Reliability } \\
\text { category }\end{array}$ \\
\hline Beam vacuum lines & 2 & 99.999995 & 4 \\
\hline Main cryogenic & 3 & 99.99999 & 3 \\
\hline \& bus-bar lines & & & \\
\hline Heat exchanger line & 1 & 99.99999 & 3 \\
\hline Auxiliary bus-bar line & 1 & 99.99998 & 2 \\
\hline Cold feet/beam & 1 & 99.99998 & 2 \\
screen cooling line & & & \\
\hline Thermal shield line & 1 & 99.99998 & 2 \\
\hline Vacuum vessel sleeve & 1 & 99.99995 & 1 \\
\hline (envelope) & & & \\
\hline
\end{tabular}

- Zone 3 - radiation and thermal shielding (C', E, N);

- Zone 4 - insulation vacuum envelope (W).

The reliability categories associated with the subsequent zones correspond to the values specified in Table 3 .

\section{Failure modes of the interconnection bellows}

The failure modes of the LHC interconnection bellows are directly related to their principal functions: continuity of the beam and insulation vacuum, transfer of helium through the pressurized channels, compensation for the thermal contraction of the cryo-magnets, stability under the pressure loads etc. The modes taken into account with respect to the LHC bellows expansion joints are grouped into 3 categories:

- material failures - comprising magnetic permeability failure, resulting from the phase transformations in stainless steel at cryogenic temperatures, and evolution of ductile damage;

- structural failures - local and global instabilities leading to excessive local deformations of the expansion joints or misalignment of the magnets;

- fatigue failures leading to propagation of fatigue cracks and causing leaks into the beam or into the insulation vacuum.

\subsection{Material failures and the relevant constitutive equations}

The material failures reflect low temperature behaviour of stainless steel comprising evolution of damage and plastic strain induced martensitic transformation $\left(\gamma \rightarrow \alpha^{\prime}\right)$. The first leads to an inadmissible increase in the density of micro-damage and to fatigue crack propagation. In its isotropic version the evolution of damage is described by the following kinetic law [3]:

$$
\dot{D}=\left(\frac{Y}{S}\right)^{a} \dot{p} H\left(p-p_{D}\right)
$$

where $D$ is the damage parameter, $p$ is the accumulated plastic strain, $Y$ denotes the strain energy density release rate, $S$ stands for the strength energy of damage and arepresents the degree of nonlinearity with respect to the damage conjugate force $Y$. Here, $H$ denotes the Heaviside function. S increases with the decreasing temperature and $a=1$ for simplicity. Both $\mathrm{S}$ and the damage threshold $p_{D}$ have to be identified at cryogenic temperatures (cf. [1]). The second phenomenon initiates the presence of the ferro-magnetic $\alpha^{\prime}$ phase in the austenitic $\gamma$ matrix. Stainless steel bellows, often used at cryogenic temperatures as compensation elements, develop strong plastic strain fields in the convolutions. Such fields contribute to the strain induced martensitic transformation that modifies the FCC $\gamma$ austenite into the BCC $\alpha^{\prime}$ martensite. The $\gamma-\alpha^{\prime}$ transformation, usually localised at crest and at root of convolutions, has an impact on the local evolution of ductile damage and fatigue life of bellows but also on the magnetic permeability of the shell. Since the $\alpha^{\prime}$ martensite is known to be ferromagnetic, a massive phase transformation may yield the structure highly susceptible to a magnetic field. This may have a crucial impact on the performance of the expansion joints applied for the construction of particle accelerators for high energy physics. Therefore, a correct choice of material, oriented towards maximum stability at cryogenic temperatures (minimum phase transformation), seems to be an important issue. The evolution of the $\alpha^{\prime}$ martensite content, induced by the plastic deformation, is described by the following kinetic law $[4,5]$ :

$$
\dot{\xi}=A\left(T, \underline{\underline{\sigma}}, \underline{\underline{\varepsilon}}^{p}\right) \dot{p} H\left(\left(p-p_{\xi}\right)\left(\xi_{L}-\xi\right)\right) \quad ; 0 \leq \xi_{L} \leq 1
$$

where $A$ is a function of the temperature, state of stress and strain rate; $p_{\xi}, \xi_{L}$ denote the phase transformation 


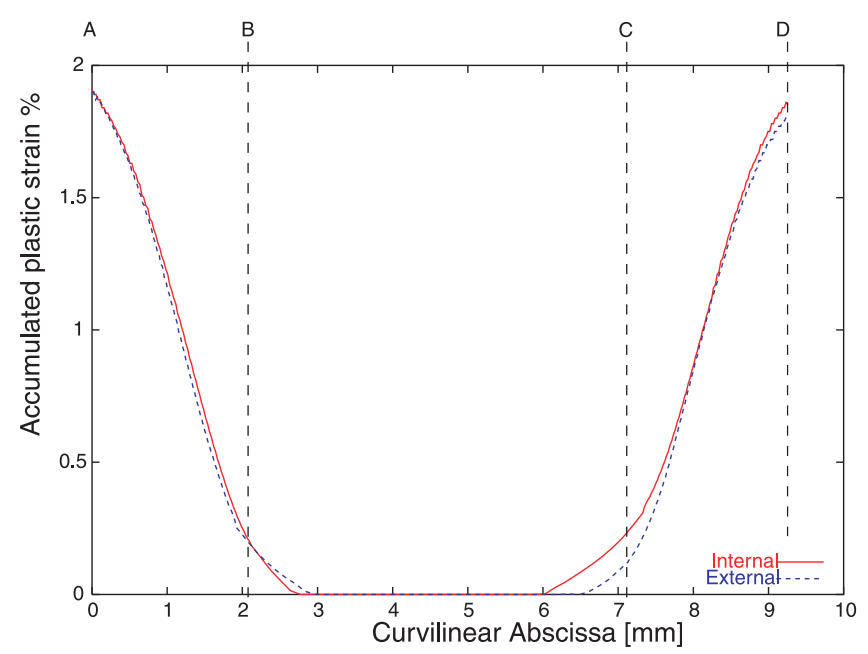

Fig. 6. Accumulated plastic strain along internal and external surface of convolution.

threshold and the saturation level, respectively. The saturation level does not necessarily correspond to the complete $(100 \%)$ transformation from $\gamma$ to $\alpha^{\prime}$ phase. Typically, for stainless steel $316 \mathrm{~L}$ this value reaches some $20 \%\left(\xi_{L}=0.2\right)$. Again, all three of them have to be identified at cryogenic conditions. Such a bi-phase material may show a very high level of magnetic permeability as a function of the plastic deformation at low temperatures. The so called magnetic failure $[6,7]$ occurs when the magnetic permeability reaches locally a predefined inadmissible level. A combination of the damage failure and the magnetic failure constitutes a criterion enabling the evaluation of the number of cycles to the generalized failure:

$$
N_{f}=F_{N}\left[\left(D-D_{c r}\right),\left(\mu(\xi)-\mu_{c r}\right)\right]
$$

where $N_{f}, \mu$ denote the number of cycles to failure and the magnetic permeability, respectively. A typical evolution of the accumulated plastic strain, damage, volume fraction of martensite and magnetic permeability along convolution is shown in Figure 6 through 9. As an example, half convolution of a typical U-type bellows has been subjected to an axial displacement cycle between $-25 \%$ and $62.5 \%$ of its initial length (- denotes compression and + denotes extension). The similarity between the diagrams 8 and 9 results from the linearity between the magnetic permeability $(\mu)$ and the martensite content $(\xi)$ for a large family of stainless steels (Fig. 12). Points A and $\mathrm{D}$ denote the root and the crest of convolution, respectively.

\subsection{Structural failures (local and global instabilities)}

The structural failures consist in the local and the global instabilities of the interconnection bellows under pressure loads $[1,8]$. These instabilities (described in terms of bifurcation buckling) are classified in the following groups:

- local wall instability (out-of-plane local buckling);

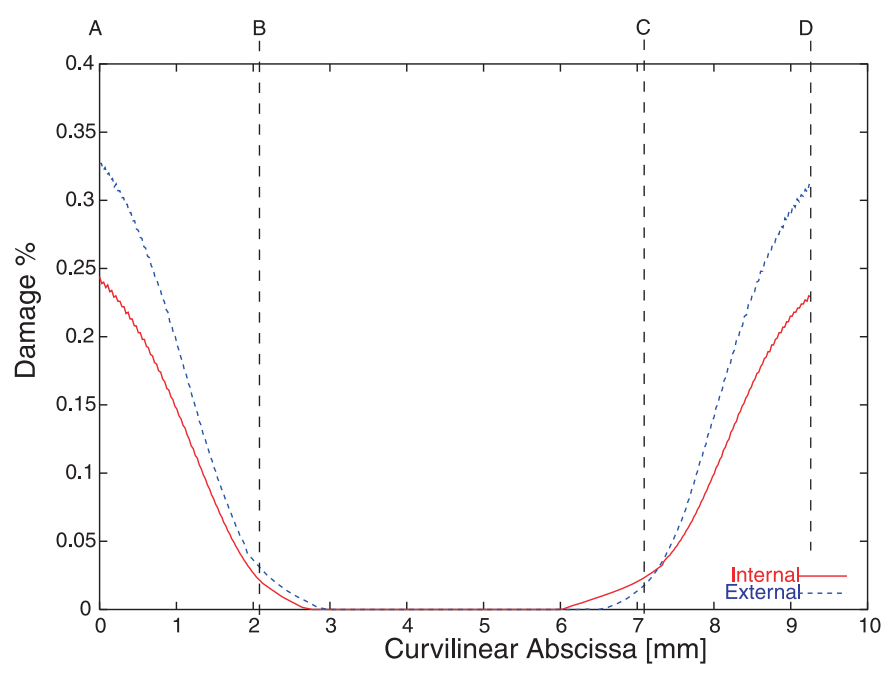

Fig. 7. Damage profile along internal and external surface of convolution.

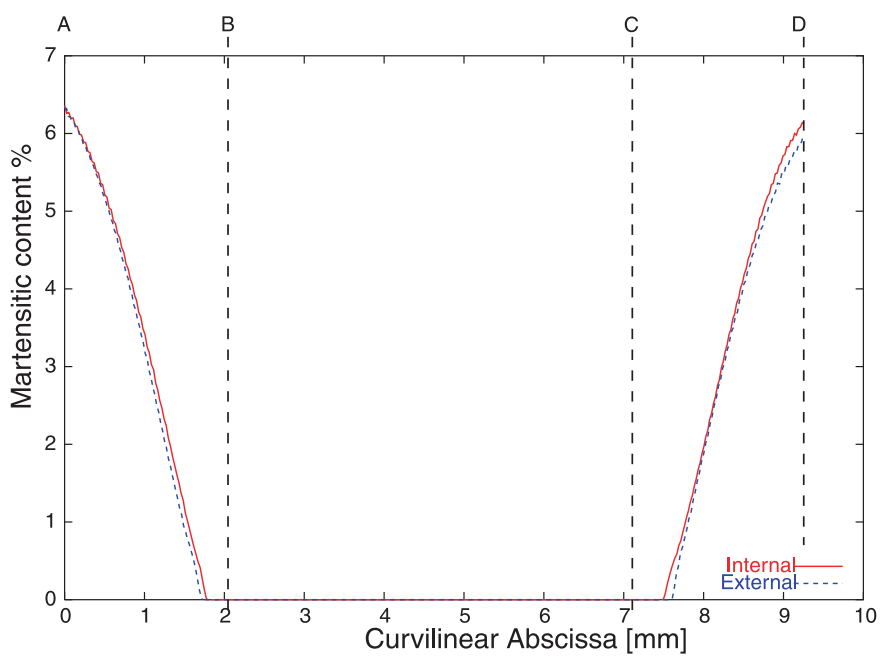

Fig. 8. Martensite content along internal and external surface of convolution.

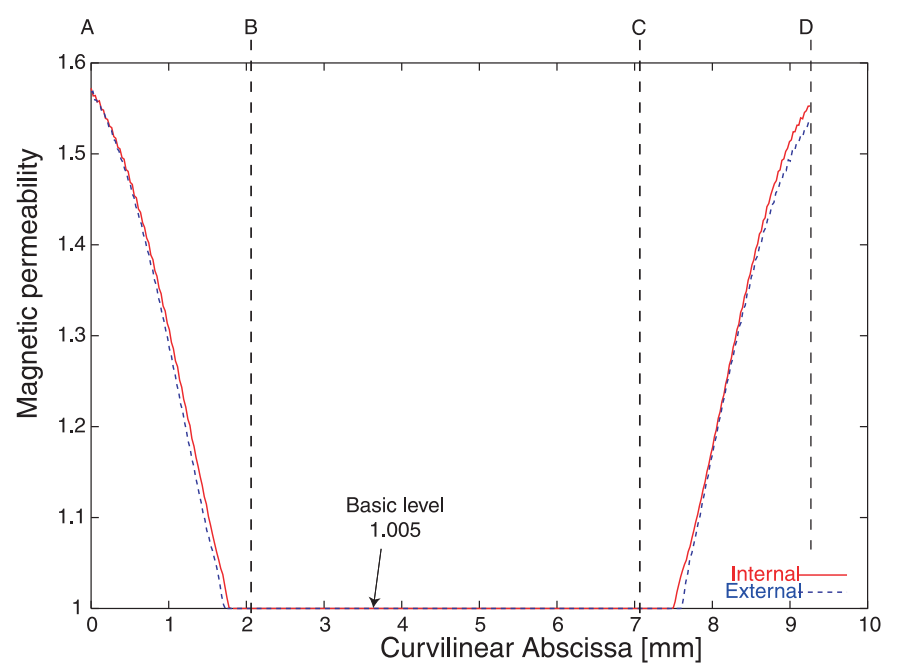

Fig. 9. Magnetic permeability along internal and external surface of convolution. 


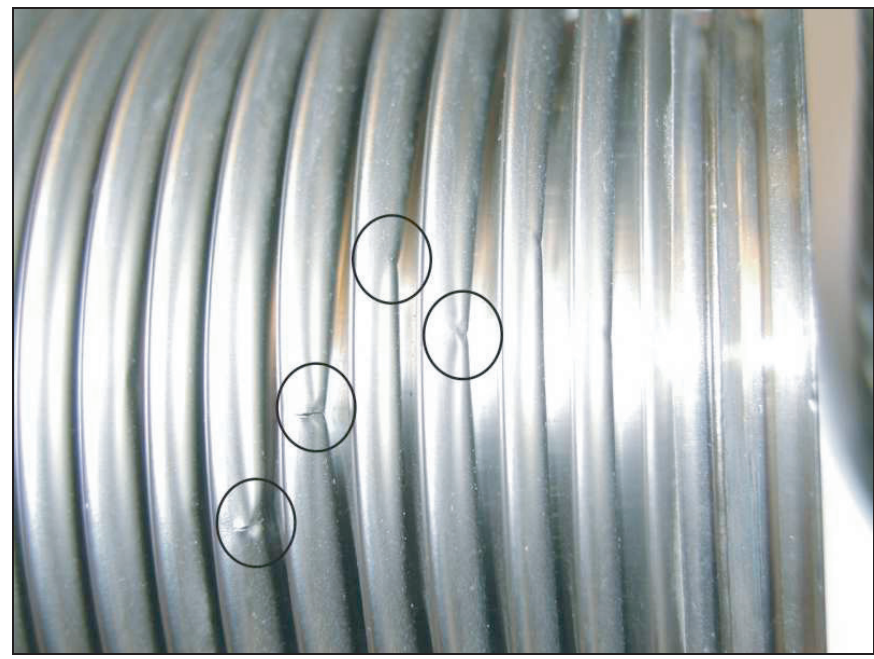

Fig. 10. Residual deformation of the shell after the local wall instability (elastic-plastic process).

- column buckling of the bellows expansion joint;

- buckling of an interconnect containing bellows expansion joint.

Local wall instabilities occur when the geometrical parameters of the convolutions and the material properties satisfy the following general criterion (cf. [9]):

$$
N_{\theta}+H^{2} N_{\phi}=\frac{2 E_{T} h^{2}}{r_{\phi} \sqrt{12\left(1-\nu^{2}\right)}}\left(1+k H^{2}\right)
$$

where $h$ denotes the wall thickness, $N_{\theta}, N_{\phi}$ are the normal circumferential and meridional forces in the mid-surface of the shell, $H=c_{\theta} / c_{\phi}$ is the ratio between the dimensions of the buckling zone and $k=r_{\phi} / r_{\theta} \quad ; r_{\theta} \neq 0, r_{\phi} \neq$ 0 is the ratio between the current local radii of curvature. $\nu$ is the Poisson coefficient. The elastic instability mode is described by the following equation:

$$
w_{t}=A \cos (m \theta) \cos (n \phi)
$$

where $w_{t}$ is the function representing the displacement field, normal to the midsurface of the shell (the LoveKirchhoff hypothesis is applied). Typical local wall instability (reaching already the elastic-plastic domain) in the convolutions of a beam vacuum bellows is shown in Figure 10.

Semi-global and global instabilities are related to the so-called column buckling of bellows and to instability of the entire interconnect, involving the environment of bellows. The analysis carried out for an equivalent column representing the bellows with its environment (neighbouring segments) leads to the eigenvalue problem (cf. $[1,10]$ ) that takes the form of the following transcendental equation:

$$
\begin{gathered}
2-\left[2+\left(\psi_{1}+\psi_{2}\right)\left(1-\gamma k^{2} L_{b}^{2}\right) k^{2} L_{b}^{2}\right] \quad \cos \left(k L_{b}\right) \\
+\left[-1+\psi_{1}+\psi_{2}+k^{2} L_{b}^{2}\left(\psi_{1} \psi_{2}+\gamma-\gamma \psi_{1} \psi_{2} k^{2} L_{b}^{2}\right)\right] \\
\times k L_{b} \sin \left(k L_{b}\right)=0
\end{gathered}
$$

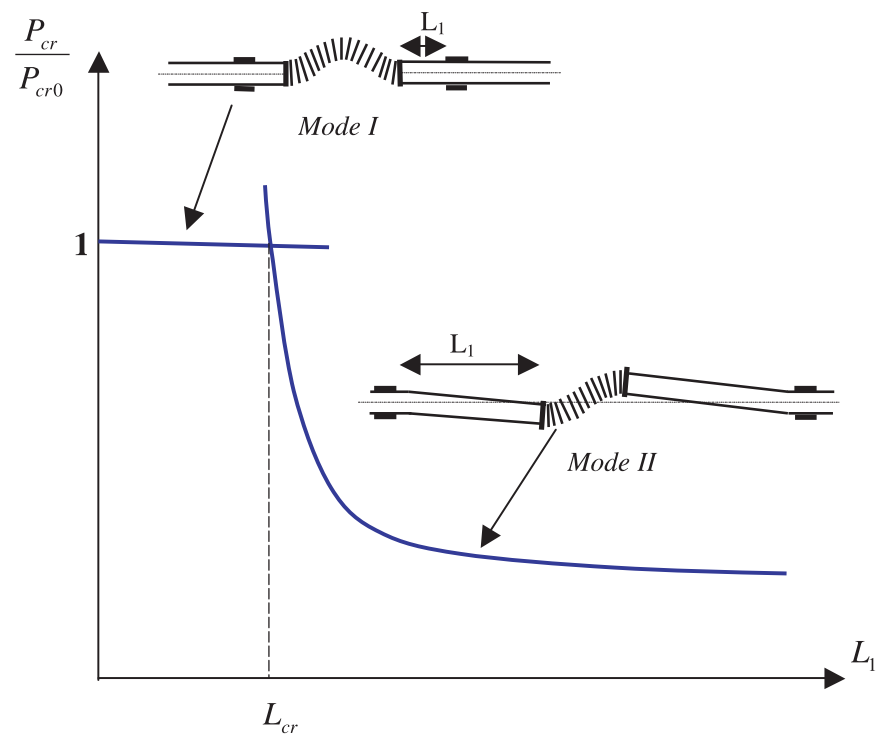

Fig. 11. Effect of guidance-to-bellows distance $\left(L_{1}\right)$ on the critical buckling pressure.

where $k L_{b}$ are the eigenvalues and the parameters $\psi_{1}, \psi_{2}, \gamma$ reflect the ratio between the bellows bending stiffness and the bending and transverse stiffness of the adjacent interconnect components. The solution leads to a stability diagram shown in Figure 11.

Two principal branches of the solution correspond to two buckling modes: buckling of bellows (mode I) and buckling of the entire system (mode II). The intersection of these two branches determines a critical guidance-tobellows distance and a transition from mode I to mode II. If the critical pressure associated with mode I turns out to be stable it is the opposite for mode II. For guidance-tobellows distance larger than the critical value the buckling pressure decreases rapidly along a very steep path to stabilise at a level much lower than the initial buckling load.

Both the local and the global instabilities may seriously compromise reliability of the interconnections and need to be carefully analysed.

\subsection{Fatigue failures}

As a result of the material failures the fatigue failures can occur. When the criterion (7) is satisfied the material is in the critical state either because of the intensity of the phase transformation or because of the intensity of the damage fields. The latter means that the conditions for the fatigue crack propagation are created. Typically, for the low-cycle fatigue at cryogenic temperatures, the fatigue criterion can be expressed by the generalized Manson-Coffin equation [11]:

$$
N_{f}^{\beta} \Delta \varepsilon_{p}\left(1-\frac{\varepsilon_{p m}}{\varepsilon_{f o}}\right)^{-\alpha \beta}=C
$$

where $\Delta \varepsilon_{p}$ is the plastic strain range and $\varepsilon_{p m}$ denotes the mean plastic strain on cycle (normalized to the tensile 
ductility). Usually, the stabilization of the stress-strain local hysteresis is reached within some 1520 cycles. Thus, all the parameters of the hysteresis $\left(\Delta \varepsilon_{p}, \varepsilon_{p m}\right)$ are measured in the stabilized configuration. Here, again the parameters $\left(\alpha, \beta, \varepsilon_{f 0}, \mathrm{C}\right)$ have to be identified at the relevant temperatures. Fatigue crack propagation through thickness leads inevitably to a diffusion of helium into the insulation vacuum and to an enhanced transport of heat by convection in the residual gas. It is worth pointing out that coupling between fatigue and stability leads to a more complex criterion limiting the life of the interconnection bellows [2]. An essential impact is attributed to the imperfection field (initial misalignment of the magnets).

\section{Combined material and structural parametric optimisation of corrugated bellows - deterministic approach}

\subsection{Optimisation of the stainless steel for cryogenic applications}

Fe-Cr-Ni austenitic stainless steels are commonly used to manufacture components of superconducting magnets and cryogenic transfer lines since they retain their ductility at low temperatures and are initially paramagnetic. Some of the nitrogen strengthened stainless steels of 300 series belong to the group of metastable austenitic alloys. Under certain conditions the steels undergo martensitic transformation at cryogenic temperatures that may lead to a considerable evolution of material properties and to a ferromagnetic behaviour. The martensitic transformations are induced mainly by the plastic strain fields and amplified by the high magnetic fields. Spontaneous transformations due to the cooling process occur in the least alloyed grades of the family such as 304L, while N-strengthened grades, such as 304LN and 316LN do not generally show spontaneous transformation during cooling.

In order to find a good compromise between the formability of the stainless steel (hydro-forming of the bellows convolutions) and the stability against the phase transformations at cryogenic temperatures the grade 316L stainless steel has been chosen. Nevertheless, in the framework of the chemical composition of the grade $316 \mathrm{~L}$ there is a room for further optimisation that might yield the material even more stable when compared to the standard market - available makes. Such optimisation requires a precise study of impact of different constituents on the intensity of martensitic transformation at cryogenic temperatures.

An increase in martensite content promoted by plastic deformation can be detected by measuring the relative magnetic permeability $\mu$. Figure 12 shows a selected reference diagram which allows estimation of the martensite content (volume fraction of martensite). It converts the susceptibility (or permeability) into martensite content. This diagram is based on the 304L, 321, and 347 SS and is accurate for magnetic measurements, performed under

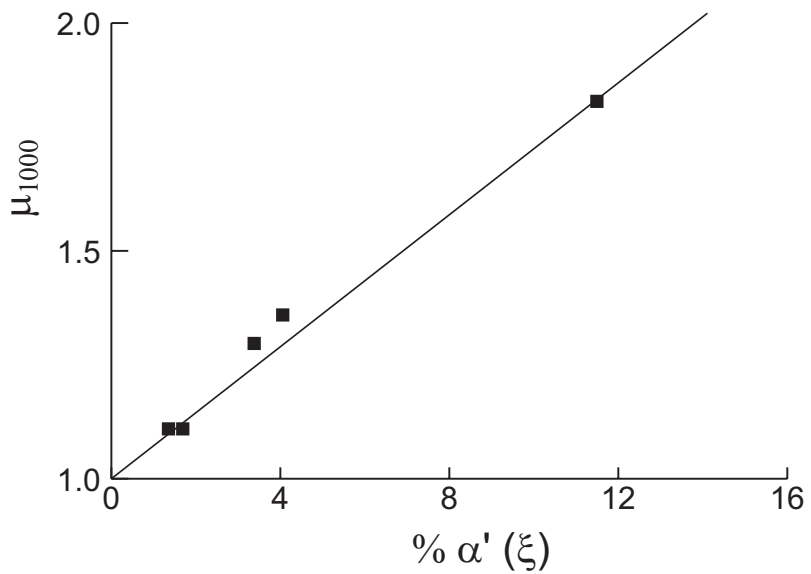

Fig. 12. Magnetic permeability versus volume fraction of $\alpha^{\prime}$ martensite for grades 304L, 321 and 347, strained at $4.2 \mathrm{~K}$ [12].

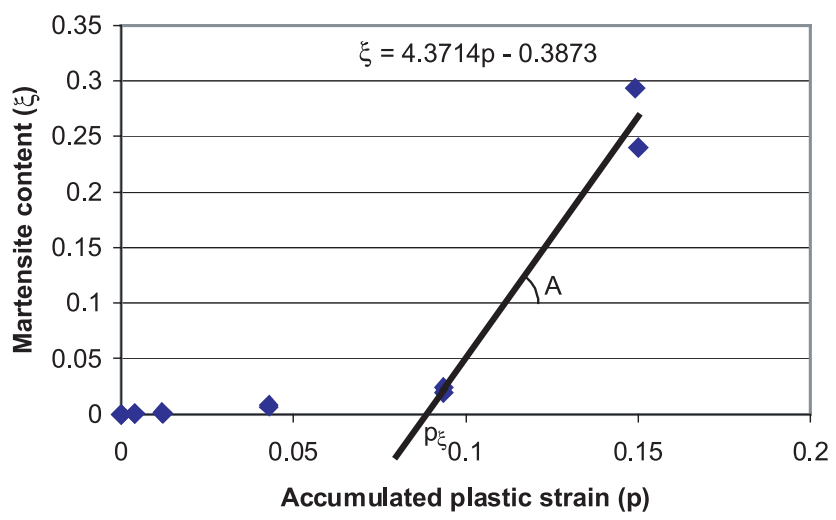

Fig. 13. Identification of parameters of Eq. (6) for grade 316L at $77 \mathrm{~K}$.

a field of 1000 Oe $(0.1 \mathrm{~T})$. Since the results for different stainless steel grades are consistent, the same diagram is considered valid for $316 \mathrm{~L}$ grade as well.

The parameters describing the phase transformation are identified from the curve of martensite content versus the inelastic strain. Typically, the volume fraction of martensite can be plotted as a function of plastic strain (Fig. 13). The slope of the line and the intersection with the $x$-axis correspond to the function $\mathrm{A}$ and the threshold $p_{\xi}$, respectively (see Eq. (6)). As an example, identification of the parameters for standard grade $316 \mathrm{~L}$ stainless steel at $77 \mathrm{~K}$ is shown in Figure 13.

Furthermore, the identification has been done for 3 temperature levels $(293,77$ and $4.2 \mathrm{~K}$ ) and the corresponding values are presented in Table 4 . In the general formulation $A$ is a function of the stress state $(\underline{\underline{\sigma}})$, strain rate $\left(\dot{\varepsilon}^{p}\right)$ and the temperature $(T)$. For the sake of simplicity $A \bar{h}$ as been identified exclusively under the total strain rate $\underline{\underline{\varepsilon}}=3.3 \times 10^{-4} \mathrm{~s}^{-1}$ and under the uniaxial stress state. More complex tests at cryogenic temperatures are planned in the future and require some further insight into the testing technique. An additional confirmation of the parameters can be obtained from the measurements of the magnetic permeability directly in the bellows convolution. 


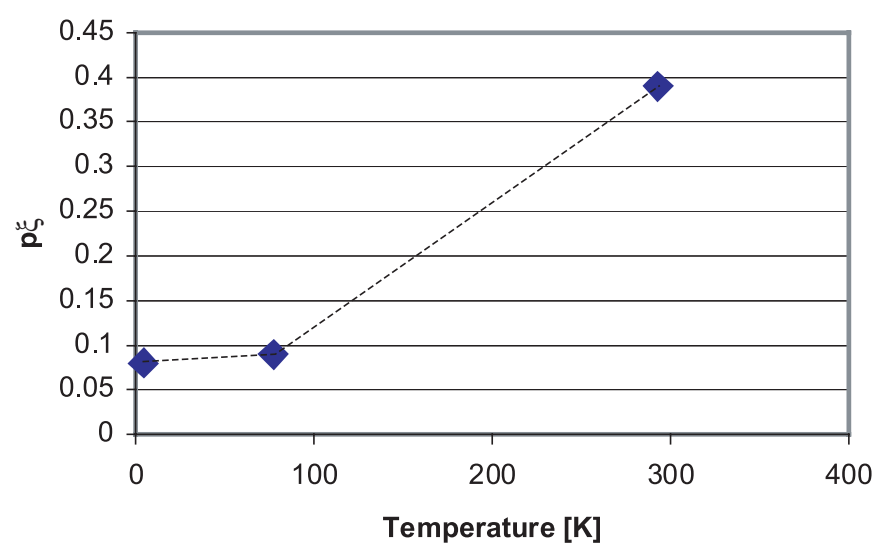

Fig. 14. Plastic strain threshold $\left(p_{\xi}\right)$ as a function of temperature.

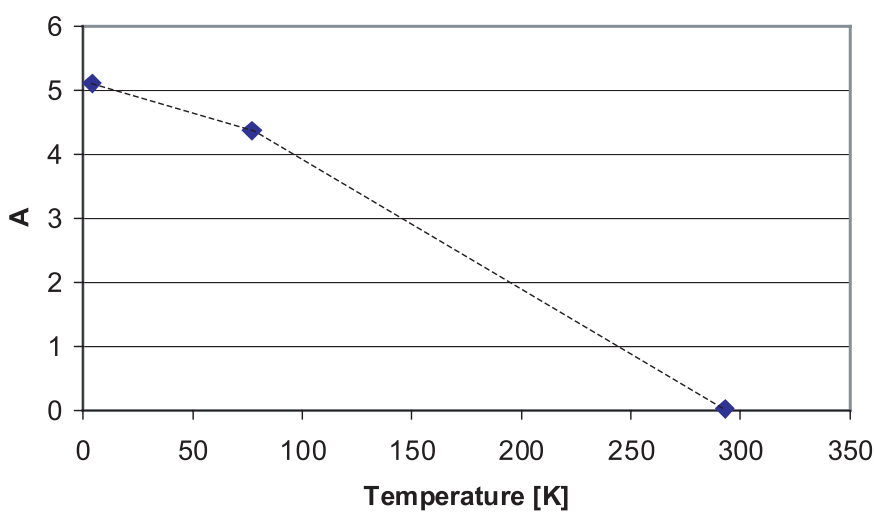

Fig. 15. Intensity parameter (A) of the martensitic transformation as a function of temperature.

Table 4. Parameters of the Eq. (6) identified at different temperatures (grade 316L).

\begin{tabular}{ccc}
\hline Temperature & $p_{\xi}$ & $A$ \\
\hline $293 \mathrm{~K}$ & 0.39 & 0.023 \\
\hline $77 \mathrm{~K}$ & 0.09 & 4.37 \\
\hline $4.2 \mathrm{~K}$ & 0.08 & 5.1 \\
\hline
\end{tabular}

The approximate evolution of two parameters describing the plastic strain induced martensitic transformation, $p_{\xi}$ and $A$ is given in Figures 14 and 15.

Optimisation of stainless steel for cryogenic applications aims at increasing the threshold $p_{\xi}$ and decreasing the slope " $A$ " (cf. Eq. (6)) in order to reduce the intensity of the phase transformation and to obtain a better stability of the material at low temperatures. An additional step that is not illustrated in the above shown plots consists in reducing the phase transformation saturation level $\xi_{L}$ (cf. Eq. (6)). Thus, the relevant general formulation of the optimisation problem is as follows:

- Maximize the objective function:

$$
F_{m}\left(p_{\xi}, A, \xi_{L}\right)=\mu_{1} \frac{p_{\xi}}{p_{\xi 0}}+\mu_{2} \frac{A_{0}}{A}+\mu_{3} \frac{\xi_{L 0}}{\xi_{L}}
$$

Table 5. Chemical composition of grade 316L for the LHC interconnection bellows.

\begin{tabular}{cc}
\hline Element & Content \\
\hline $\mathrm{C}$ & 0.024 \\
\hline $\mathrm{Si}$ & 0.49 \\
\hline $\mathrm{Mn}$ & 1.77 \\
\hline $\mathrm{P}$ & 0.015 \\
\hline $\mathrm{S}$ & 0.001 \\
\hline $\mathrm{Cr}$ & 17.41 \\
\hline $\mathrm{Ni}$ & 13.69 \\
\hline $\mathrm{Mo}$ & 2.76 \\
\hline $\mathrm{N}$ & 0.075 \\
\hline
\end{tabular}

where $p_{\xi 0}, A_{0}, \xi_{L 0}$ are the reference values and the following condition is satisfied for the weight functions:

$$
\mu_{1}+\mu_{2}+\mu_{3}=1
$$

- Constraints:

grade $316 \mathrm{~L}$ stainless steel $\Rightarrow$ chemical composition;

- Design variables:

parameters of the chemical structure $\eta_{N i}, \eta_{M o}, \eta_{C r}, \eta_{N}$. In the first simplified approach, only the minimization of the $\xi_{L}$ (maximization of $\frac{\xi_{L 0}}{\xi_{L}}$ ) has been performed. Different grades of 316L stainless steel were tested at low temperatures. By modifying mainly the nickel content (one parameter of the chemical structure) a local minimum of $\xi_{L}$ was obtained and the following final material (special grade) has been chosen for the interconnection bellows of the LHC (Tab. 5): form.

The material has been manufactured in a very similar

\subsection{Structural parametric optimisation of corrugated bellows}

Optimum design of the corrugated bellows for the LHC interconnections has been carried out by adopting some of the EJMA stress and stability evaluation formulas (cf. [13]). However, all the aspects related to cryogenic conditions (material properties, fatigue curves, etc.) have been redefined and introduced into the specially conceived optimisation procedure. The main objective of the parametric optimisation [1] is to minimise the bellows axial stiffness (Eq. (3)). Usually the available space constitutes one of the major geometrical constraints. Generally, we search for minimum axial stiffness under the geometrical, stress, stability and fatigue constraints. The relevant formulation of the optimisation problem is given below:

- minimise the objective function:

$$
F_{a x}\left(\underline{x}_{b l}\right) ;
$$


- equality constraints:

$$
\text { inner diameter } \quad D_{\text {in }}=\text { const.; }
$$

- inequality constraints:

$\begin{array}{ll}\text { bellows convoluted length } & L_{b l} \leq L_{\max } \\ \text { outer diameter } & D_{\text {out }} \leq D_{\max } \\ \text { bellows maxi compression } & \Delta_{b l-} \geq \Delta_{\min } \\ \text { cost function } & Q_{b l} \leq Q_{0 b l} \\ \text { membrane stress } & S_{1} \leq S_{a d} \\ \text { membrane stress } & S_{2} \leq S_{a d} \\ \text { membrane \& bending stress } & S_{34} \geq\left(S_{3}+S_{4}\right) \\ \text { fatigue life } & N_{f}\left(S_{t}\right) \geq N_{f 0} \\ \text { column buckling } & P_{c o l} \geq P_{1} \\ \text { in-plane squirm } & P_{i n p} \geq P_{2}\end{array}$

- design variables:

$$
\underline{x_{b l}} \rightarrow\left(n_{c}, n_{p}, t_{p}, q, w, \zeta\right)
$$

where $\zeta=\Delta_{+} / \Delta_{-}$is the ratio between the bellows extension and compression, and $q$ stands for the convolution pitch. $S_{t}$ is the EJMA equivalent stress. $Q_{b l}$ denotes the real cost of the bellows, technologically justified.

A simplified parametric way of solving the problem is based on the algorithm where the number of design variables is reduced to 4 :

$$
\underline{x_{b l}} \rightarrow\left(n_{c}, t_{p}, q, w\right)
$$

The number of plies $n_{p}$ is defined at the beginning of the procedure and the parameter $\zeta=\Delta_{+} / \Delta_{-}$is recomputed from the admissible compression $\Delta_{b l-}$ (inequality constraint).

The algorithm searches for all the solutions, for which the axial stiffness is smaller than a check value:

$$
F_{a x} \leq F_{a x}^{v}
$$

arbitrarily chosen at the beginning of the process. The local minimum solutions are collected and a global minimum is chosen for a given number of plies. The operation can be repeated for different number of plies and, finally, a global minimum is found. The optimisation domain (between the constraint lines) has been discretized and the objective function checked point-by-point. A typical form of the design objective, plotted against wall thickness and the number of convolutions (for a given constant convoluted length), with the fatigue, strength and stability constraints is shown in Figure 16. As a matter of fact, the degree of nonlinearity in this process is not very high therefore the analysis is rather fast and not CPU time consuming. The advantage of the above presented algorithm of sweeping directly the objective function in the optimisation domain consists mainly in the possibility of finding always the global minimum without the additional cost of verifying the necessary and sufficient conditions. For the LHC bellows expansion joints the solution was usually found on the stability constraint because
Table 6. Fatigue life test results for nested bellows.

\begin{tabular}{cc}
\hline Number of test & Number of cycles to failure \\
\hline 1 & 1520 \\
\hline 2 & 1586 \\
\hline 3 & 1640 \\
\hline 4 & 1760 \\
\hline
\end{tabular}

reduction of the bellows axial stiffness reduces simultaneously the bifurcation buckling load (column buckling mode). In some cases, however, the domain of optimisation was reduced to a small "island" between the fatigue and strength/stability constraints and both strength and stability conditions were activated.

\section{Reliability and cost oriented optimisation - probabilistic approach}

\subsection{Survival probability of bellows}

In order to illustrate analysis of the survival probability of the LHC interconnection components two examples are chosen: the nested bellows and the RF-contact bellows expansion joints (both integrated into the beam vacuum interconnections, Fig. 4). The "survival probability" is defined as the probability that the unit will survive 50 thermo-mechanical cycles needed for the lifetime of the LHC.

The fatigue tests carried out at room temperature reveal a scatter of the number of cycles to failure, under the same test conditions, related to material and geometrical imperfections. The Weibull probability density function has been introduced [14] in order to describe the probability of fatigue failure. The Weibull distribution generates the failure rate that varies linearly with time (or number of cycles). The cumulative distribution function is defined for $N$ cycles by the following formula:

$$
P_{F}(N)=1-\exp \left(-\left(\frac{N}{\delta}\right)^{\lambda}\right)
$$

where $\delta$ is the localisation parameter and $\lambda$ stands for the shape parameter. Identification of both parameters by means of the Nelson-Thompson diagram for a typical set of data (Tab. 6) for nested bellows [15] is shown in Figure 17.

The survival probability:

$$
R(N)=1-P_{F}(N)
$$

as a function of the number of cycles is plotted in Figure 18. Similar analysis has been carried out for the second compensation unit in the beam vacuum interconnections - the RF-contact bellows [15]. The corresponding survival probability diagram is shown in Figure 19.

Both diagrams indicate the survival probability of the components of the beam vacuum interconnections (w.r.t. 

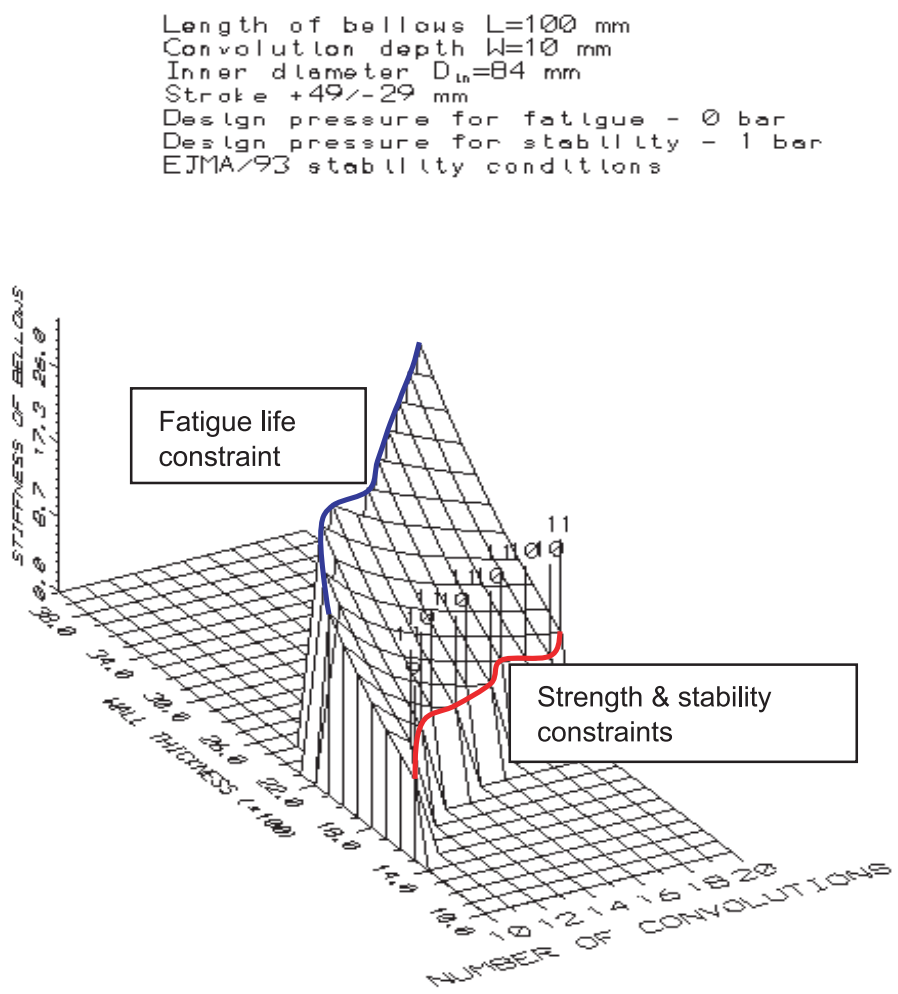

Fig. 16. The local minimum solutions in the optimisation procedure.

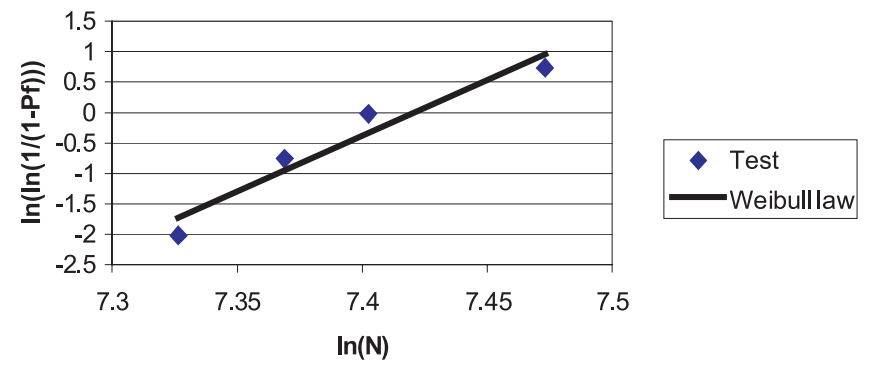

Fig. 17. Identification of parameters of the Weibull function for the nested bellows.

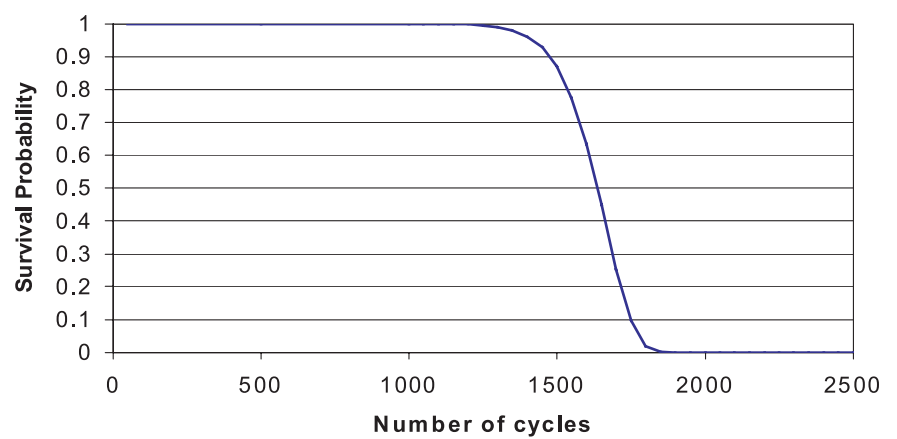

Fig. 18. Survival probability as a function of the number of cycles for nested bellows at $293 \mathrm{~K}$.

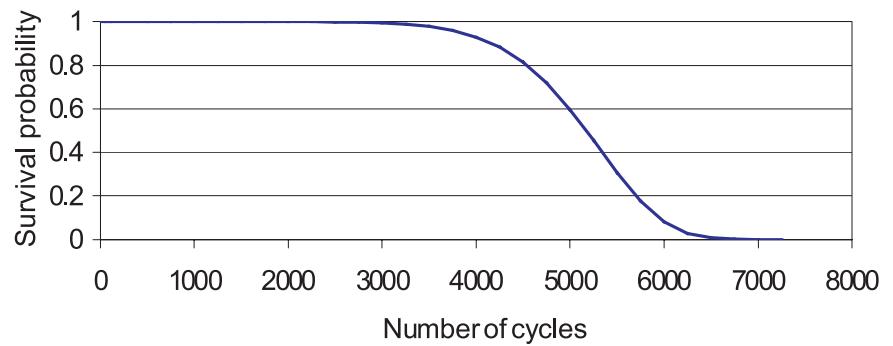

Fig. 19. Survival probability for RF-contact bellows at $293 \mathrm{~K}$.

the required 50 cycles) above the specified values (see Tab. 7).

Accelerated life testing provides a basis for definition of a more general function, useful in the optimisation of bellows:

$$
R=R\left(n_{c}, t_{p}, q, w\right)
$$

This function reflects the influence of basic design parameters on the reliability of bellows. Hence, for a given bellows geometry it is possible to predict its survival probability, with respect to the required number of cycles. 
Table 7. Reliability of the Nested and RF-contact bellows for 50 cycles.

\begin{tabular}{cll}
\hline Type of bellows & Apportionned reliability level & Measured reliability (w.r.t. 50 cycles) \\
\hline Nested bellows & $99.999999 \%$ & $99.99999999 . . \%$ \\
\hline RF bellows & $99.999999 \%$ & $99.9999998 \%$ \\
\hline
\end{tabular}

\subsection{Optimisation algorithm - semi-probabilistic approach}

The semi-probabilistic approach constitutes the first step towards the reliability oriented parametric optimisation. The formulation of the optimisation problem remains similar to the formulation presented in the Section 5.2. The difference consists in replacing the condition:

$$
N_{f}\left(S_{t}\right)-N_{f 0} \geq 0
$$

by the equation:

$$
R\left(N_{0}\right)-R_{0} \geq 0
$$

where $N_{0}, R_{0}$ denote the required number of cycles (target value) and the apportioned reliability level, respectively. Thus, the fatigue life constraint is replaced by the reliability constraint. It is worth pointing out that the optimum solution is usually located close to the strength and stability constraints (see Fig. 16), on the boundary of the optimisation domain. Since the above explained modification of the optimisation problem will have an impact on the upper constraint line (fatigue $\rightarrow$ reliability), it will mainly influence the size of the optimisation domain. Thus, the majority of the optimum solutions will remain the same, with the exception of the situations where the optimisation domain is considerably reduced and both constraint lines intersect each other.

\subsection{Optimisation algorithm - fully probabilistic approach}

The fully probabilistic approach [1] consists in searching for minimum axial stiffness under the geometrical, stress, stability and fatigue constraints - everything in the framework of the previously apportioned reliability. The relevant formulation of the optimisation problem is given below:

- minimise the objective function:

$$
F_{a x}\left(\underline{x}_{b l}\right) ;
$$

- equality constraints:

$$
\text { inner diameter } \quad D_{\text {in }}=\text { const.; }
$$

- inequality constraints: deterministic

$$
\begin{array}{ll}
\text { bellows convoluted length } & L_{b l} \leq L_{\max } \\
\text { outer diameter } & D_{\text {out }} \leq D_{\max } \\
\text { bellows maxi compression } & \Delta_{b l-} \geq \Delta_{\min } \\
\text { cost function } & Q_{b l} \leq Q_{0 b l}
\end{array}
$$

probabilistic

$\begin{array}{ll}\text { probability of failure } & \left.P(\underline{x})\right|_{\Omega_{b l}} \leq p_{0 b l} \\ \text { membrane stress } & P\left[S_{1}>S_{a d}\right] \leq p_{2 b l} \\ \text { membrane stress } & P\left[S_{2}>S_{a d}\right] \leq p_{2 b l} \\ \text { membrane } & \\ \text { \& bending stress } & P\left[S_{3}+S_{4}>S_{34}\right] \leq p_{2 b l} \\ \text { fatigue life } & P\left[N_{f}\left(S_{t}\right)<N_{f 0}\right] \leq p_{3 b l} \\ \text { column buckling } & P\left[P_{\text {col }}<P_{1}\right] \leq p_{4 b l} \\ \text { in-plane squirm } & P\left[P_{\text {inp }}<P_{2}\right] \leq p_{4 b l}\end{array}$

- random design variables:

$$
\underline{x_{b l}} \rightarrow\left(n_{c}, n_{p}, t_{p}, q, w, \Delta_{+} / \Delta_{-}\right)
$$

Here $\Delta_{+} / \Delta_{-}$denotes the repartition of the axial offset between compression and tension and $S_{t}$ stands for the EJMA equivalent stress.

Thus, the objective is to minimise the axial stiffness under the equality constraint of constant inner diameter, deterministic inequality constraints concerning the geometry and the cost function as well as the probabilistic constraints defined as follows:

- overall probability of failure $P(\underline{x})$ for the bellows is limited to the predefined value $p_{0 b l}$;

- probability of the stresses $S_{1}, S_{2}, S_{3}+S_{4}$ being grater than the predefined values $S_{a d}, S_{34}$, is limited to $p_{2 b l}$;

- probability of number of cycles to failure $N_{f}$ being smaller than the predefined value $N_{f 0}$, is limited to $p_{3 b l}$

- probability of the critical pressures for the column buckling and the in-plane squirm being smaller than the predefined values $P_{1}, P_{2}$, is limited to $p_{4 b l}$.

This formulation requires more knowledge about the probability density functions corresponding to different phenomena like the stress levels, fatigue failure and the stability issues. For the time being, the probability density functions related to the fatigue life were identified for some of the components. Assuming that the family of Weibull functions is applicable, the parameters can be identified as a function of the vector $\underline{x}$ :

$$
P_{F}(N, \underline{x})=1-\exp \left(-\left(\frac{N}{\delta(\underline{x})}\right)^{\lambda(\underline{x})}\right)
$$

Thus, it is possible - for a given technological process - to create a database with two functions: $\delta(\underline{x}), \lambda(\underline{x})$. In the process of optimisation both functions shall be available as multi-dimensional tables.

For what concerns the probability density functions related to the mechanism of local or global instability 
the applicable family is closer to the normal distribution since the critical loads depend mainly on the geometrical imperfections. Thus, the probability density function can be expressed as follows:

$$
P_{F}(N, \underline{x})=\frac{1}{\sigma \sqrt{2 \pi}} \exp \left(-\frac{1}{2}\left(\frac{N-m(\underline{x})}{\sigma(\underline{x})}\right)^{2}\right)
$$

with $m(\underline{x})$ and $\sigma(\underline{x})$, the mean and the standard deviation respectively, as the tables for the optimisation process. These tables can be found by testing a number of components per given geometry $(\underline{x})$ against instability and by applying a standard identification process for the parameters of normal distribution.

Similar procedure can be adopted for the EJMA type stresses that depend on the modulus of elasticity at a given temperature, on the bellows geometry and on the loads. Assuming both the material properties and loads identical for all the samples, the stresses are again function of geometrical imperfections and normal probability density function can be applied.

Finally, overall probability of failure is related to magnetic failure and damage evolution. A combined criterion based on damage parameter and magnetic permeability can be applied. Both functions can be measured by using micro-Vickers technique and by using magnetometers. Again for each given geometry $(\underline{x})$ the parameters of the relevant probability density function have to be established. In order to minimise the cost of the above specified tests and operations, the numerical simulations can be extremely useful (Monte-Carlo method).

\section{Discussion and conclusions}

The present paper highlights a number of important issues in the design of compensation systems, containing bellows expansion joints, for the super-conducting particle accelerators. The bellows - thin-walled axi-symmetric shell structures - are installed in the magnet-to-magnet interconnection space in order to provide continuity of beam vacuum, cryogenic channels and to compensate for the thermal contraction/expansion of the magnets. As a part of the beam vacuum chamber they belong to the so called beam transport system and constitute its drift (non-magnetic) parts. The bellows are subjected to intensive plastic straining already during the phase of hydroforming, that belongs to the manufacturing process. In the interconnections the bellows are subjected to large axial deformations at cryogenic temperatures accompanied by high pressures and distortions resulting from transverse and angular misalignments. Since one of the principles in the design of colliders for high energy physics is maximisation of the magnetic length (active length of all the main dipoles and quadrupoles), the non-magnetic drift space in the interconnections has to be minimised. This implies a strong optimisation of the bellows expansion joints in terms of their geometry (volume) and in terms of their performance. The first direct consequence is the fact that the LHC interconnection bellows work in the low-cycle fatigue regime. High intensity plastic strain fields lead to local phase transformations and to development of damage fields. One of the important issues in the optimum design of bellows is a combination of the material and structural optimisation. The present paper presents such approach where the first part of the optimisation algorithm consists in increasing the stability of stainless steel at low temperatures against the martensitic transformation. Here a new grade 316L stainless steel has been developed in order to maximise a function based on the transformation parameters. The second part of the design is based on the reliability oriented structural optimisation where the material, stability and fatigue failures are taken into account. Typical design objective in the optimisation process is the axial flexibility that has to be maximised under strength, stability reliability (fatigue) and geometrical constraints. The constraints can be expressed either in deterministic or probabilistic way. It is worth pointing out that both the material and the structural optimisation contribute to the final success of the collider in terms of its reliability.

The following conclusions can be formulated as a consequence of the approach presented in the paper:

- Successful operation of the LHC interconnections over the scheduled period of 20 years depends very much on the reliability of all the structural components, in particular the bellows expansion joints;

- Corrugated bellows belong to the most critical components of cryogenic compensation systems and are subjected to extremely sever service conditions;

- Optimum design oriented towards the reliability of bellows consists of two principal parts: optimum choice of the material (stainless steel) and reliability oriented structural optimisation;

- Design of stainless steel aims at maximising its stability at cryogenic temperatures. A new alloy has been found in the framework of the grade $316 \mathrm{~L}$ that satisfies this requirement;

- The main objective of the structural optimisation is to minimise the bellows stiffness under geometrical, strength, stability and reliability (fatigue) constraints;

- The reliability levels achieved in the process of optimisation of the LHC bellows expansion joints shall correspond to the previously apportioned reliability levels to all the systems and sub-systems of the LHC interconnections;

- The solution of the structural optimisation for most of the LHC interconnection bellows has been found close to the stability constraint;

- Optimisation of a bellows should not compromise the stability of the whole interconnect, therefore a stability diagram has to be developed for each interconnection line. Also a global stability of the collider under the pressure loads has to be computed once the local design of the compensation system is completed;

The present paper certainly opens many perspectives of further research and a study leading to a more complete formulation of the optimisation problem is currently being developed. 


\section{References}

[1] B. Skoczen, Stability, fatigue and optimization of thinwalled structures under cryogenic conditions. Application in the structural design of colliders and cryogenic transfer lines., CERN Yellow Report, CERN-2001-001, Geneva (2001)

[2] B. Skoczen, Local stability of cryogenic pipelines and cryomagnet interconnections - effect of fatigue damage evolution on buckling, ASME PVP2000 Conference, Seattle, published in PVP-Vol. 401, ASME (2000)

[3] J. Lemaitre, A Course on Damage Mechanics, SpringerVerlag (1992)

[4] C. Garion, B. Skoczen, Plastic strain induced damage evolution and martensitic transformation in ductile materials at cryogenic temperatures, Proceedings of the International Cryogenic Materials Conference, ICMC (2001), Madison, USA, Advances in Cryogenic Engineering, Vols. 47 and 48, New York, (2001)

[5] C. Garion, B. Skoczen, Modeling of plastic strain induced martensitic transformation for cryogenic applications, ASME J. Appli. Mech. 69 (2002)

[6] K. Suzuki, J. Fakakura, H. Kashiwaya, H., Cryogenic fatigue properties of $304 \mathrm{~L}$ and $316 \mathrm{~L}$ stainless steels compared to mechanical strength and increasing magnetic permeability, J. Test. Evaluation 16

[7] M.L. Delsante, C. Garion, S. Sgobba, B. Skoczen, Experimental analysis of the plastic strain induced martensitic transformation in 316L stainless steel at cryogenic temperatures and identification of the relevant constitutive equations, CERN Technical Note, EDMS $n^{\circ} 411336$

[8] B. Skoczen, Local stability of cryogenic pipelines and cryomagnet interconnections - generalised equivalent column concept, ASME PVP2000 Conference, Seattle, published in PVP-Vol. 401, ASME (2000)

[9] E.L. Axelrad, Flexible Shells, Moskva, Nauka in Russian (1976)

[10] B. Skoczen, Effect of Shear Deformation and Relaxation of Support Conditions on Elastic Buckling of Pressurised Expansion Bellows, ASME Journal of Pressure Vessel Technology, 121 (1999), pp. 127-132

[11] B. Skoczen, Generalisation of the Coffin equations with respect to the effect of large mean plastic strain, ASME Journ. Eng. Mat. Techn., 118, July (1996)

[12] D.C. Larbalestier, H.W. King, Austenitic stainless steels at cryogenic temperatures - Part I: structural stability and magnetic properties, Cryogenics 13, 160-167 (1973)

[13] EJMA STANDARDS, Standards of the Expansion Joint Manufacturers Association, Inc., New York, USA, (1995)

[14] I. Kovalenko I., N.Y. Kuznetsov, Ph.A. Pegg, Mathematical theory of reliability of time depend systems with practical applications, John Wiley \& Sons Ltd., (1997)

[15] C. Garion,B. Skoczen, Reliability oriented optimum design of the LHC interconnections. Part I: mechanical compensation system, LHC Project Note 245, EDMS 249743, CERN (2000) 